\title{
Producción de biodiésel por etanolisis utilizando aceites de fritura de hoteles y su uso en calderas pirotubulares
}

\section{Biodiesel production by ethanolysis using waste cooking oil from hotels for firetube boilers}

\author{
Edwin Alberto Bulla-Pereira \\ M. Sc. Ingeniero mecánico. \\ Universidad ECCI. \\ Bogotá, Colombia \\ ebullap@ecci.edu.co
}

\author{
Carlos Alberto Guerrero-Fajardo \\ Ph. D Ingeniería Química y Química Catálisis. \\ Universidad Nacional de Colombia. \\ Bogotá, Colombia \\ caguerrerofa@unal.edu.co
}

\author{
Fabio Emiro Sierra-Vargas \\ Ph. D. Ingeniería Mecánica. \\ Universidad Nacional de Colombia. \\ Bogotá, Colombia \\ fesierrav@unal.edu.co
}

Resumen- La producción de biocombustibles a partir del aceite usado de fritura (AUF) es una importante alternativa para utilizarse en la generación de biodiésel, además su uso contribuiría en la reutilización de un producto altamente contaminante. Este trabajo busca determinar el mayor rendimiento del proceso de producción de ésteres etílicos a partir de los parámetros: relación molar AUF/etanol (1:5 - 1:7), porcentaje de catalizador en peso $(0,5-0,7 \% w t)$, temperatura de reacción $\left(50-60^{\circ} \mathrm{C}\right.$ ) y como agente de lavado (agua a 40 ${ }^{\circ} \mathbf{C}$ - ácido acético). El proceso de producción se realizó en dos etapas, la primera es una esterificación con catálisis homogénea ácida (H2SO4) y, la segunda, de transesterificación con catálisis alcalina $(\mathrm{NaOH})$. El proceso utiliza una temperatura de reacción de $60^{\circ} \mathrm{C}$, relación molar AUF: etanol de 1:7 y agua como agente de lavado. El biodiésel producido posee características fisicoquímicas según los norma ASTM D 6751, y un rendimiento de ésteres etílicos de ácidos grasos (FAEE) del 93,5\%. Las condiciones óptimas encontradas en el biocombustible permiten el uso en "calderas" que funcionan con diésel.

Palabras clave- Aceites usados de fritura, biodiésel, esterificación, transesterificación.

Abstract- Biofuel production from waste cooking oil (WCO) is an important alternative for using in generating biodiesel also it helps in the reuse of a pollutant. This work seeks to determine the best performance of the production process of ethyl esters from the parameters: molar ratio WC0/ethanol (1: 5-1: 7), percentage of catalyst by weight $(0.5$ to $0.7 \% w t)$, reaction temperature $\left(50-60{ }^{\circ} \mathrm{C}\right)$ and washing agent $\left(40{ }^{\circ} \mathrm{C}\right.$ Water - acetic acid) using the transesterification process by homogeneous catalysis alkaline $(\mathrm{NaOH})$. The process was carried out in two steps, the first stage of esterification and other one transesterification with a reaction temperature of $60^{\circ} \mathrm{C}$, molar ratio WCO: ethanol 1: 7 and water as washing agent. The biodiesel produced possesses physicochemical characteristics according to ASTM D 6751 standard and $93.5 \%$ yield of ethyl esters. The optimal conditions enable the use in biofuel "boiler" which functional diesel.

Keywords- Oils for frying, biodiesel, esterification, transesterification.

\section{INTRODUCCIÓN}

Actualmente el $81 \%$ de la energía mundial consumida es proporcionada por combustibles de origen fósil, el petróleo aporta el 32,4\%, el carbón el $27,3 \%$ y el gas natural provee el $21,4 \%$. El sector del transporte emplea el $57,7 \%$ de la energía producida actualmente en el mundo. El consumo y aumento en el empleo de los combustibles fósiles en los diferentes sectores económicos está creando grandes cantidades de gases efecto invernadero. En las dos últimas décadas se produjeron 32 Gt/año de $\mathrm{CO}_{2}$ [1], además de otros gases tipo invernadero ( $\mathrm{NOx}, \mathrm{CO}, \mathrm{PM}, \mathrm{HC}, \mathrm{O}_{3}, \mathrm{CH}_{4}$ ) que generan graves problemas medio ambientales [2], y afectan especialmente el calentamiento global, según pronósticos para el 2040 el sector industrial crecerá anualmente un 1,8\% [3], al considerarse una demanda total de energía en el mundo para este mismo año de 20664 Mtoe [1]. Este consumo au- 
mentará los problemas del medio ambiente debido al volumen de producción de los gases arrojados a la atmósfera del planeta. Para satisfacer la demanda de energía y disminuir las dificultades ambientales se requiere un mayor uso, investigación y desarrollo de las energías renovables y, en especial, de los biocombustibles, debido a la utilización de estos en varios sectores económicos de cada país. El biodiésel es una excelente fuente de energía renovable que contribuye con la mitigación en las emisiones de los gases efecto invernadero y contribuye con la disminución de la dependencia hacia los combustibles fósiles. En la Fig. 1 se observa la reducción porcentual de las emisiones de gases tipo invernadero a partir del empleo de biodiésel.

Fig. 1. REDUCCIÓN PORCENTUAL DE EMISIONES DE GASES TIPO INVERNADERO

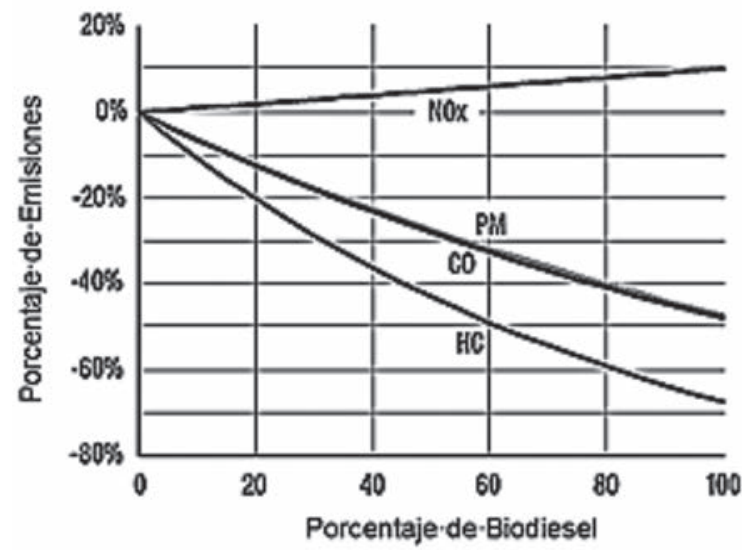

Fuente: [4].

La Sociedad Americana de Ensayos y Materiales (ASTM) define al biodiésel como ésteres monoalquílicos de ácidos grasos de cadena larga derivados de insumos grasos renovables, como los aceites vegetales o grasas animales [5] o aceites usados de fritura (AUF). Actualmente se producen 154 millones t/año en el mundo [6]. Este biocombustible tiene propiedades fisicoquímicas similares al diésel, aspecto que permite el uso en motores de combustión interna, calderas u otros equipos industriales que empleen el combustible fósil como fuente de energía. La producción del biodiésel emplea el procedimiento de la esterificación o transesterificación. (1) Reacción de transesterificación que se genera en la producción del biodiésel.

$$
\mathrm{RCOOR}^{1}+\mathrm{R}^{2} \mathrm{OH} \stackrel{\text { Catalizador }}{\longleftrightarrow} \mathrm{RCOOR}+\mathrm{R}^{1} \mathrm{OH}
$$

El proceso de transesterificación se puede efectuar por una reacción sin catalizador (condiciones súper críticas para el metanol) y una reacción catalizada [7] que emplea para su proceso algunos de los tres tipos de catalizadores: heterogéneo, enzimático u homogéneo [8]. El proceso transesterificación en reacción catalizada junto con los catalizadores homogéneos se emplea actualmente en la producción industrial. Estos procesos se pueden obtener de manera discontinua o batch (lotes) o de forma continua [9]. La producción promedio del biodiésel en Colombia es, en la actualidad, de 44214 t/mes [10]. Producción relativamente baja con respecto a pronósticos de demanda requerida en el país. Estos aspectos mencionados hacen viable realizar una investigación en la determinación del mayor rendimiento del proceso de producción de ésteres etílicos, establecido en las mejores condiciones de producción utilizando el proceso de transesterificación de AUF de hoteles mediante hidróxido de sodio $(\mathrm{NaOH})$. Con las máximas condiciones de la producción de biodiésel se diseña y construye una planta de producción en proceso tipo Batch y el biocombustible obtenido se emplea en una caldera pirotubular, con el fin de obtener datos que permitan establecer características en el uso del biocombustible en equipos industriales.

\section{MATERIALES}

\subsection{Reactivos}

La calidad de los reactivos es un factor que afecta el rendimiento del proceso y calidad del producto. Para este trabajo se emplearon los siguientes reactivos: etanol $\left(\mathrm{C}_{2} \mathrm{H}_{6} \mathrm{O}_{2}\right)$ con el 96\% de pureza (alquímicos), $\mathrm{NaOH}$ en escamas con una pureza del 98\% (alquímicos), ácido sulfúrico $\left(\mathrm{H}_{2} \mathrm{SO}_{4}\right)$ del $98 \%$ (químicos Campota), ácido acético $\left(\mathrm{C}_{2} \mathrm{H}_{4} \mathrm{O}_{2}\right)$ glacial del $99 \%$ (alquímicos) y aceite usado de fritura de soja (AUFS) suministrado por el sector hotelero.

\section{DISEÑO EXPERIMENTAL}

\subsection{Producción de biodiésel}

La producción de los FAEE se plantea a partir de la ecuación (2). El catalizador empleado es $\left(\mathrm{H}_{2} \mathrm{SO}_{4}\right)$ para la etapa de esterificación y $\mathrm{NaOH}$ 
para la etapa de transesterificación. En este estudio la variable de temperatura de reacción se establece en 50 y $60{ }^{\circ} \mathrm{C}\left(\mathrm{X}_{3}\right)$. La cantidad de etanol necesaria se establece a partir de la relación molar AUFS:etanol de 1:5 y 1:7 $\left(\mathrm{X}_{1}\right)$. Un porcentaje en peso de catalizador $(0,5-0,7)\left(X_{2}\right)$ es disuelto en el etanol. Esta mezcla es vertida en $200 \mathrm{~mL}$ AUFS filtrado previamente. El porcentaje del rendimiento se determina con la ecuación (2) [11].

$$
\text { Rendimiento } \%=\frac{\text { Peso de biodiesel }}{\text { Peso de AUFS }} \times 100
$$

Los experimentos se llevan a cabo a escala de laboratorio. Las reacciones se producen en un reactor enchaquetado de $500 \mathrm{~mL}$ en vidrio con cuatro bocas, equipado con un condensador lineal, un termómetro, un agitador magnético y un baño termostático con controlador. El reactor se mantiene a la temperatura de reacción, una velocidad de agitación de 200 RPM, a presión atmosférica y tiempo de reacción de $1 \mathrm{~h}$. Finalizado el tiempo se adiciona al reactor un volumen de ácido acético agitándolo por 15 min. La solución obtenida es vertida a un embudo separador de $500 \mathrm{~mL}$ a temperatura ambiente por un tiempo de $12 \mathrm{~h}$. Los productos obtenidos por el proceso de decantación (biodiésel y glicerina) son depositados cada uno en embudo de $500 \mathrm{~mL}$. Para la purificación y lavado del biocombustible producido se emplearon dos agentes de lavado (agua a $40{ }^{\circ} \mathrm{C}$ - ácido acético) $\left(\mathrm{X}_{4}\right)$.

\subsection{Proceso de optimización}

La optimización del proceso de transesterificación se lleva a cabo a través de un experimento con 4 factores, en el cual se examinan los efectos que tienen los parámetros relación molar (RM) de aceite/etanol, temperatura de reacción (T), porcentaje de catalizador (\%p/p C) y el agente de lavado (AL). Para la determinación del rendimiento de ésteres de etilo se empleó un diseño central compuesto (DCC). El DCC consta de 31 unidades experimentales con las cuales se debe obtener la información suficiente para permitir un modelo polinómico de segundo orden. El punto axial tuvo un $\alpha=1$. Los valores centrales (nivel cero) elegidos para el nivel DCC fueron: relación molar aceite/etanol 1/6, concentración de catalizador $0,6 \%$, temperatura de reacción de $55^{\circ} \mathrm{C}$ y agua a temperatura ambiente como agente de lavado. La Tabla I muestra los niveles y el símbolo de codificación utilizados para cada factor. Para evitar los errores experimentales se efectuaron las corridas experimentales en orden aleatorio como se muestra en la Tabla I. El software empleado fue Minitab 16 en el que se obtuvieron la regresión y gráfico de los datos adquiridos.

Los datos experimentales presentados en la Tabla I se analizan mediante el procedimiento de regresión de superficie de respuesta (RSR). El procedimiento RSR utiliza un análisis canónico para estimar los valores para cada uno de los factores [12]. Usando el modelo ajustado de la superficie de respuesta se construyen gráficas para cada par de factores que se estudian mientras se mantiene el factor del rendimiento constante en su punto estacionario estimado.

\subsection{Análisis de FAEE con cromatografía de gases}

Los FAEE se analizaron usando cromatografía de gases (CG). El equipo empleado es marca Hewlett Packard Serie II referencia 5890. La temperatura del inyector fue de $350{ }^{\circ} \mathrm{C}$ y la del detector fue 390 ${ }^{\circ} \mathrm{C}$. El gas portador fue nitrógeno con un flujo de 8 $\mathrm{mL} / \mathrm{min}$. Los datos son obtenidos con el programa Cerity QA/QC versión A.04.05.

\subsection{Planta piloto de biodiésel}

Para desarrollar la planta piloto de biodiésel es necesario determinar el mayor rendimiento del proceso de producción de ésteres etílicos basado en las mejores condiciones de operación obtenidas en las pruebas de laboratorio. El proceso de la planta para su desarrollo y construcción fue de tipo Batch, con una capacidad de 150 L/Lote. El montaje se efectúo en el Laboratorio de Biocombustibles del Departamento de Química de la sede Bogotá de la Universidad Nacional de Colombia. La etapa experimental en la planta se desarrolló a partir de varias fases: Análisis del balance de masa por operación del proceso de producción, selección de equipos, desarrollo y planteamiento de la planta, está última fase se desarrolló en tres partes. La primera, construcción y diseño de un diagrama de flujo del proceso (PFD). La segunda, fue la caracterización física de los equipos de la planta para realizar los planos de despiece, montaje y explosivo del diseño en el espacio asignado para el montaje. La última etapa consistió en la construcción y puesta a punto de la planta piloto para la implementación en la producción de biodiésel con los parámetros establecidos en el laboratorio. 
TABLA I

MATRIZ DEL DISEÑO CENTRAL, COMPUESTO DE LAS CUATRO VARIABLES CON SUS SÍMBOLOS DE CODIFICACIÓN, NIVELES Y \% DE LOS RENDIMIENTOS

\begin{tabular}{|c|c|c|c|c|c|}
\hline Orden de Corrida & $\mathrm{X}_{1} ;(\mathrm{RM})$ & $\mathrm{X}_{2} ;(\% \mathrm{p} / \mathrm{p} \mathrm{C})$ & $\mathrm{X}_{3} ;(\mathrm{T})$ & $\mathrm{X}_{4} ;(\mathrm{AL})$ & \% Rendimiento \\
\hline 1 & $-1 ;(1: 5)$ & $0 ;(0,6)$ & $0 ;(55)$ & 0 ; (Agua) & 79,6 \\
\hline 2 & $1 ;(1: 7)$ & $1 ;(0,7)$ & $1 ;(60)$ & $1 ;\left(\right.$ Agua a $\left.40^{\circ} \mathrm{C}\right)$ & 88,1 \\
\hline 3 & $0 ;(1: 6)$ & $1 ;(0,7)$ & $0 ;(55)$ & 0 ; (Agua) & 85,7 \\
\hline 4 & $0 ;(1: 6)$ & $0 ;(0,6)$ & $0 ;(55)$ & 0 ; (Agua) & 87,9 \\
\hline 5 & $0 ;(1: 6)$ & $0 ;(0,6)$ & $0 ;(55)$ & 0 ; (Agua) & 88,1 \\
\hline 6 & $1 ;(1: 7)$ & $-1 ;(0,5)$ & $1 ;(60)$ & -1 ; (Ácido acético) & 74,4 \\
\hline 7 & $0 ;(1: 6)$ & $-1 ;(0,5)$ & $0 ;(55)$ & 0 ; (Agua) & 85,7 \\
\hline 8 & $0 ;(1: 6)$ & $0 ;(0,6)$ & $0 ;(55)$ & $1 ;\left(\right.$ Agua a $\left.40^{\circ} \mathrm{C}\right)$ & 77,3 \\
\hline 9 & $-1 ;(1: 5)$ & $1 ;(0,7)$ & $-1 ;(50)$ & 1 ; (Agua a $\left.40^{\circ} \mathrm{C}\right)$ & 78,6 \\
\hline 10 & $-1 ;(1: 5)$ & $1 ;(0,7)$ & $1 ;(60)$ & $1 ;\left(\right.$ Agua a $\left.40^{\circ} \mathrm{C}\right)$ & 74,3 \\
\hline 11 & $-1 ;(1: 5)$ & $-1 ;(0,5)$ & $-1 ;(50)$ & $1 ;$ (Agua a $\left.40^{\circ} \mathrm{C}\right)$ & 76,8 \\
\hline 12 & $0 ;(1: 6)$ & $0 ;(0,6)$ & $0 ;(55)$ & 0 ; (Agua) & 88,5 \\
\hline 13 & $1 ;(1: 7)$ & $-1 ;(0,5)$ & $-1 ;(50)$ & $1 ;\left(\right.$ Agua a $\left.40^{\circ} \mathrm{C}\right)$ & 80,1 \\
\hline 14 & $0 ;(1: 6)$ & $0 ;(0,6)$ & $0 ;(55)$ & 0 ; (Agua) & 88,9 \\
\hline 15 & $-1 ;(1: 5)$ & $-1 ;(0,5)$ & $1 ;(60)$ & $-1 ;\left(\right.$ Agua a $\left.40^{\circ} \mathrm{C}\right)$ & 80,8 \\
\hline 16 & $-1 ;(1: 5)$ & $-1 ;(0,5)$ & $1 ;(60)$ & $1 ;\left(\right.$ Agua a $\left.40^{\circ} \mathrm{C}\right)$ & 78,9 \\
\hline 17 & $0 ;(1: 6)$ & $0 ;(0,6)$ & $0 ;(55)$ & 0 ; (Agua) & 75,6 \\
\hline 18 & $0 ;(1: 6)$ & $0 ;(0,6)$ & $1 ;(60)$ & 0 ; (Agua) & 81,9 \\
\hline 19 & $1 ;(1: 7)$ & $-1 ;(0,5)$ & $-1 ;(50)$ & -1 ; (Ácido acético) & 78,5 \\
\hline 20 & $1 ;(1: 7)$ & $1 ;(0,7)$ & $1 ;(60)$ & -1; (Ácido acético) & 80,0 \\
\hline 21 & $1 ;(1: 7)$ & $1 ;(0,7)$ & $-1 ;(50)$ & -1 ; (Ácido acético) & 71,9 \\
\hline 22 & $-1 ;(1: 5)$ & $1 ;(0,7)$ & $-1 ;(50)$ & -1 ; (Ácido acético) & 84,6 \\
\hline 23 & $0 ;(1: 6)$ & $0 ;(0,6)$ & $0 ;(55)$ & 0 ; (Agua) & 75,2 \\
\hline 24 & $1 ;(1: 7)$ & $0 ;(0,6)$ & $0 ;(55)$ & 0 ; (Agua) & 79,8 \\
\hline 25 & $0 ;(1: 6)$ & $0 ;(0,6)$ & $-1 ;(50)$ & 0 ; (Agua) & 81,8 \\
\hline 26 & $-1 ;(1: 5)$ & $-1 ;(0,5)$ & $-1 ;(50)$ & -1; (Ácido acético) & 74,1 \\
\hline 27 & $0 ;(1: 6)$ & $0 ;(0,6)$ & $0 ;(55)$ & 0 ; (Agua) & 89,1 \\
\hline 28 & $0 ;(1: 6)$ & $0 ;(0,6)$ & $0 ;(55)$ & -1 ; (Ácido acético) & 77,5 \\
\hline 29 & $1 ;(1: 7)$ & $-1 ;(0,5)$ & $1 ;(60)$ & $1 ;\left(\right.$ Agua a $\left.40^{\circ} \mathrm{C}\right)$ & 80,7 \\
\hline 30 & $1 ;(1: 7)$ & $1 ;(0,7)$ & $-1 ;(50)$ & $1 ;\left(\right.$ Agua a $\left.40^{\circ} \mathrm{C}\right)$ & 68,5 \\
\hline 31 & $-1 ;(1: 5)$ & $1 ;(0,7)$ & $1 ;(60)$ & -1 ; (Ácido acético) & 76,8 \\
\hline
\end{tabular}

\subsection{Experimentación en caldera pirotubular}

La experimentación se realiza en una caldera vertical pirotubular ubicada en los laboratorios del patio de mecánica de la Universidad Nacional de Colombia (Fig. 2). No obstante, anteriormente a las pruebas en caldera se realizaron otras pruebas con el biocombustible obtenido en la planta piloto. Una de estas fue la prueba a la llama, para esto se emplea un mechero Bunsen, un soporte de acero, un triángulo de porcelana y un crisol. Otra prueba fue la evaluación de la combustión con el quemador de la caldera, en la cual se analizan el consumo, la eficiencia energética y las emisiones producidas a cinco muestras de biodiésel 
obtenido en la planta piloto. Los parámetros analizados fueron: volumen de biocombustible alimentado, tiempo de encendido del quemador y análisis de gases de la combustión. Experimentación que hace posible observar el comportamiento del quemador de la caldera al emplearse una cantidad determinada de diésel comercial (B10) y una de biodiésel producido en la planta piloto (B100). Para las pruebas se construyó un equipo con una estructura en acero, recubierto en su interior por ladrillos refractarios y en un extremo se acopló el quemador de la caldera vertical pirotubular. El quemador tiene un motor bifásico de $110 \mathrm{~V} / 60 \mathrm{~Hz}$ de 0,5 HP, con un sistema de alimentación de aire de 3450 RPM y una ignición mediante chispa eléctrica a $10000 \mathrm{~V}$. Este pulveriza el combustible para generar un proceso de combustión con llama no premezclada. Durante las pruebas realizadas se emplea un flujo de aire igual a $0,123 \mathrm{~m} 3 / \mathrm{s}$, el consumo de combustible para una mezcla B10 es igual a 0,342 L/min y para el B100 es igual a $0,282 \mathrm{~L} / \mathrm{min}$. (Fig. 2).

Fig. 2. CALDERA PIROTUBULAR VERTICAL (IZQ) - BANCO DE PRUEBAS DE COMBUSTIÓN (DER)
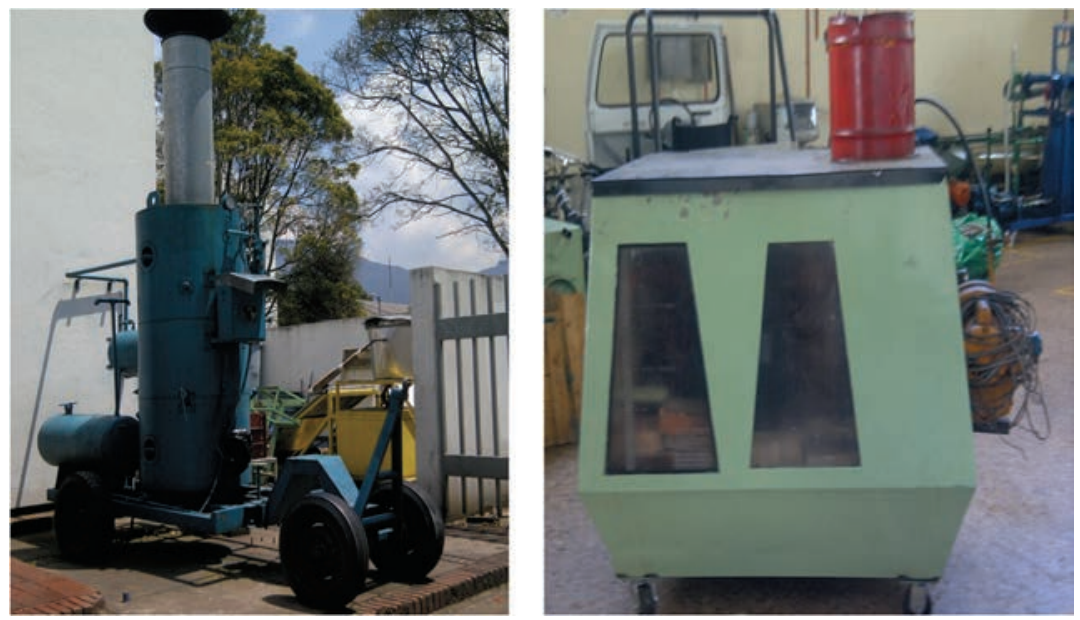

Fuente: autores.

\section{RESULTADOS}

\subsection{Proceso de transesterificación en dos etapas}

Pruebas de índice de acidez (IA) a muestras del AUFS dieron como resultado un promedio de 7,65 $\mathrm{mg} \mathrm{KOH/g}$ aceite. Este resultado determina una proporción muy alta de AGL en el aceite. La literatura reporta que una materia prima (aceite) ideal tiene un $\mathrm{VA}<1 \mathrm{mg} \mathrm{KOH} / \mathrm{g}$ aceite aunque investigaciones establecen una tolerancia entre $(<0,5-2,2 \% p / p)$. Este exceso de AGL en el AUFS plantea una alta condición de hidrolisis [13]. Este establece que para reducir estas proporciones de AGL se debe aplicar un proceso de esterificación al AUFS para reducir la reacción de neutralización de los AG o la reacción de saponificación [14]. A partir de la ecuación 2 se constituye que en la etapa de esterificación el rendimiento de la fase esterificada es cercano al $70 \%$ y en la segunda etapa del proceso de transesterificación, la concentración de ésteres etílicos de ácidos grasos es

de $90 \% \mathrm{p} / \mathrm{p}$ y un $10 \% \mathrm{p} / \mathrm{p}$ del glicerol con otros compuestos. El mejor rendimiento obtenido de las unidades experimentales fue del $88,9 \%$, logrado en el laboratorio a partir de las condiciones de operación de relación molar aceite:alcohol de 1:7, $\% \mathrm{p} / \mathrm{p}$ catalizador de 0,7 , temperatura de $60^{\circ} \mathrm{C}$ y agente de lavado agua a $40^{\circ} \mathrm{C}$.

\subsection{Análisis del método de superficie de respuesta de la transesterificación}

La transesterificación mediante catálisis homogénea alcalina se realiza utilizando el pretratamiento de las muestra con un índice de acidez $4,3 \mathrm{mg}$ de $\mathrm{KOH} / \mathrm{g}$. Con el fin de buscar las condiciones óptimas para la síntesis de biodiésel, los experimentos se realizan de acuerdo con la matriz de DCC (Tabla I). Las respuestas observadas de la conversión de biodiésel se muestran también en la Tabla I. A partir del análisis de regresión múltiple de los datos experimentales se obtiene la siguiente ecuación polinómica de segundo orden (3). 
$Y=83,74+0,55 X_{1}+0,21 X_{2}+1,85 X_{3}-$ $0,43 X_{4}-2,86 X_{1}^{2}+3,14 X_{2}^{2}-0,71 X_{3}^{2}-5,16 X_{4}^{2}+$ $0,22 X_{1} X_{2}+2,05 X_{1} X_{3}+0,93 X_{1} X_{4}+1,42 X_{2} X_{3}-$ $1,56 X_{2} X_{4}+0,61 X_{3} X_{4}$

Donde $\mathrm{Y}$, es la respuesta, es decir, la conversión a biodiésel, de las variables codificadas $X_{1}$,

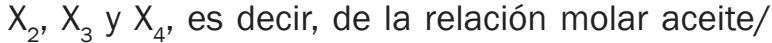
etanol, concentración de catalizador, tiempo de reacción y temperatura, respectivamente.

El análisis de la varianza (ANOVA) de los resultados del modelo de superficie de respuesta revela que este modelo es adecuado para expresar la relación real entre la respuesta y las variables planteadas, con un coeficiente de determinación $\left(\mathrm{R}^{2}=\right.$ 0,8842 ), lo que indica que el $88 \%$ de la variabilidad en la respuesta puede ser explicada por la ecuación polinómica de segundo orden establecida (3). Los perfiles de las superficies de respuesta de la producción óptima de rendimiento basado en la ecuación 3, se muestran en la Fig. 3, con los parámetros fijos de tiempo de reacción de $1 \mathrm{~h}$, velocidad de agitación de 200 rpm y presión atmosférica. La Fig. 3-A muestra que la cantidad de catalizador es muy importante en relación con la temperatura de reacción en la producción del biodiésel a partir de AUF, porque el mayor rendimiento se observa a 0,7 $\% \mathrm{p} / \mathrm{p}$ de catalizador y una temperatura de $60^{\circ} \mathrm{C}$, ya que cualquier cantidad por debajo muestra una disminución en el rendimiento del biodiésel. La Fig. 3-B representa la interacción entre la relación molar AUFS:etanol y el agente de lavado. El mejor rendimiento del producto se consigue en una relación molar 1:6 y agua como agente de lavado, obteniéndose un rendimiento aproximado del $83 \%$ en peso. La interacción de la relación molar AUFS:etanol en la producción del biodiésel se presenta en la Fig. 3-C, esta muestra que la máxima relación molar y la máxima temperatura planteada en el estudio son factores que tienen efectos significativos en el porcentaje del rendimiento. La superficie de la Fig. 3-D establece que hay una interacción mutua entre el agente de lavado (agua) y la temperatura a $60{ }^{\circ} \mathrm{C}$, definiendo que la temperatura más adecuada está alrededor de $60^{\circ} \mathrm{C}$. El aumento de la producción del FAEE se favorece cuando se emplea un alto porcentaje de catalizador; sin embargo, relaciones molares menores a 1:6 con concentraciones de catalizador en el punto central $(0,5 \% p / p)$ dan lugar a la producción de emulsiones que dificulta la separación de las fases, disminuyendo la conversión alrededor del 82\%, como se observa en la Fig. 3-E. El rendimiento obtenido alrededor del $87 \%$ aproximadamente de biocombustible se logra con el mayor porcentaje de catalizador $(0,7 \% p / p)$ y el nivel central empleado en la variable del agente de lavado, porque con los factores empleados por debajo de los parámetros mencionados anteriormente se obtienen rendimientos por debajo del $75 \%$ de producción del combustible; como se puede observar en la superficie de la Fig. 3. Los valores óptimos de las variables de prueba en las unidades experimentales son los siguientes: Relación molar AUFS/etanol 1:7, \%p/p catalizador de 0,7; temperatura de reacción de $60{ }^{\circ} \mathrm{C}$; agua como agente de lavado, con el correspondiente $\mathrm{Y}=88,9 \%$.

\section{3 Análisis de las propiedades del biodiésel}

Las composiciones de las muestras de biodiésel son analizadas por cromatografía de gases. Los datos obtenidos del cromatograma muestran que el 96,4\% de la composición del biodiésel obtenido son ésteres etílicos de ácidos grasos (FAEE), estableciendo que los triglicéridos (aceite) reaccionaron para formar FAEE, MG y DG. De acuerdo con la norma EN 14105 y ASTM D6584 el contenido máximo de MG, DG y TG presente en el biodiésel debe ser 0,8\%, 0,2\%, 0,2\%, respectivamente [15]. Los resultados muestran que el contenido de MG no cumple con el parámetro, porque supera en $2,8 \%$ al estándar de la norma, aspecto que se refleja por la presencia de agua en el biodiésel, debido a tiempos muy cortos en el proceso de separación entre las fases, pero si los DG y TG cumplen las condiciones de normatividad. Otras de las propiedades evaluadas en el combustible se establecen en la Tabla II.

\section{4 Planta piloto}

El proceso que se empleará en la planta para su desarrollo y construcción es de tipo Batch, esta tendrá una capacidad de 150 L/Lote. El montaje se efectuará en el Laboratorio de Biocombustibles del Departamento de Química de la sede Bogotá de la Universidad Nacional de Colombia. A partir de las variables obtenidas en el proceso de optimización que se efectuó el diseño de la planta. La Fig. 3 muestra el diseño de montaje realizado en el software Solidworks y el montaje de la planta en el laboratorio (Fig. 4). El diseño del equipo se estableció con siete subsistemas (Tabla III). 
Fig. 3. TRAZADO DE SUPERFICIES DE RESPUESTA DEL PORCENTAJE EN PESO DEL RENDIMIENTO DEL BIODIÉSEL OBTENIDO ENTRE LOS EFECTOS EN LA: CONCENTRACIÓN DEL CATALIZADOR VS TEMPERATURA (A); RELACIÓN MOLAR AUFS/ETANOL VS AGENTE DE LAVADO (B), RELACIÓN MOLAR AUFS/ETANOL VS TEMPERATURA (C), TEMPERATURA VS AGENTE DE LAVADO (D), RELACIÓN MOLAR AUFS/ETANOL VS CONCENTRACIÓN DE CATALIZADOR (E), CONCENTRACIÓN DE CATALIZADOR VS AGENTE DE LAVADO (F)

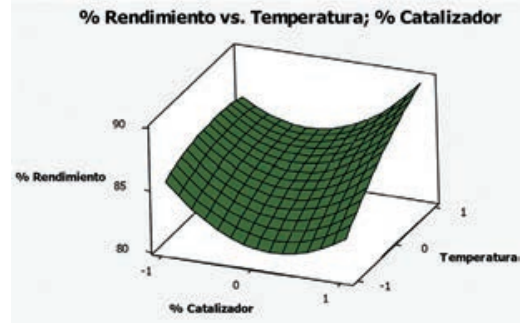

(a)

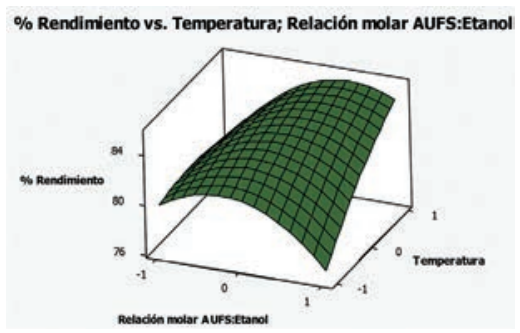

(c)

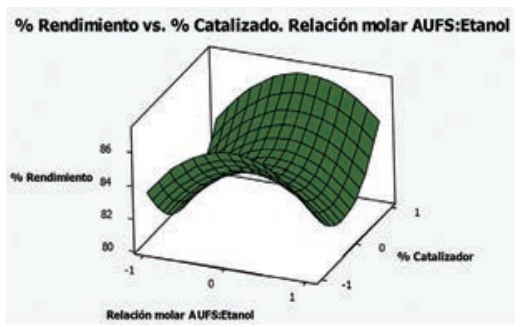

(e)

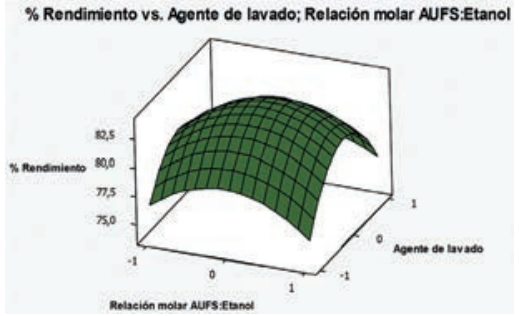

(b)

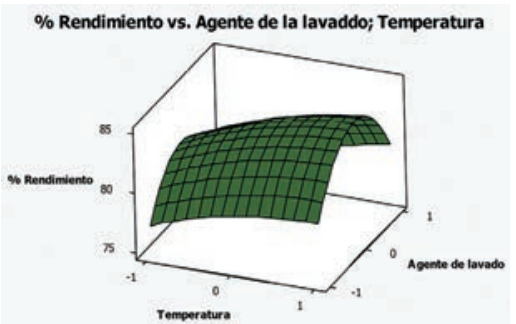

(d)

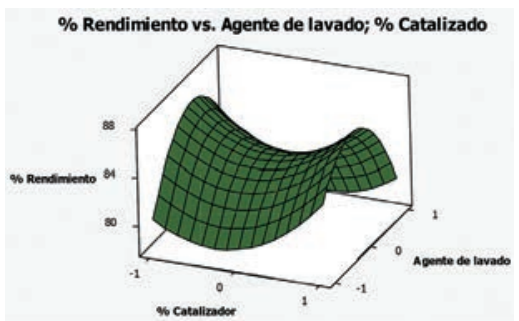

(f)

Fuente: autores.

TABLA II

PROPIEDADES DEL BIODIÉSEL OBTENIDO CON LAS MEJORES CONDICIONES DE OPERACIÓN

\begin{tabular}{|l|c|c|c|}
\hline \multicolumn{1}{|c|}{ Parámetro } & Valor obtenido & Unidad & Norma \\
\hline Densidad a $15^{\circ} \mathrm{C}$ & 899 & $\mathrm{~kg} / \mathrm{m}^{3}$ & EN ISO 12185 \\
\hline Viscosidad a $40^{\circ} \mathrm{C}$ & 4,45 & $\mathrm{~mm} / \mathrm{s}$ & ASTM D445 \\
\hline Contenido de cenizas & 0,057 & $\%(\mathrm{~m} / \mathrm{m})$ & ASTM - D 6751 \\
\hline Índice de acidez & 0,39 & $\mathrm{mg} \mathrm{KOH} / \mathrm{g}$ aceite & EN 14104 \\
\hline Punto de nube & 4,0 & ${ }^{\circ} \mathrm{C}$ & ASTM-D 2500 \\
\hline Contenido de agua & 0,056 & $\mathrm{~g} / \mathrm{mol}$ & ASTM-D 130 \\
\hline Masa molar & 303,15 & $\%(\mathrm{~m} / \mathrm{m})$ & {$[16]$} \\
\hline Monoglicéridos & 3,59 & $\%(\mathrm{~m} / \mathrm{m})$ & EN14105 \\
\hline Diglicéridos & 0 & $\%(\mathrm{~m} / \mathrm{m})$ & EN14105 \\
\hline Triglicéridos & 0 & & EN14105 \\
\hline
\end{tabular}


TABLA III

SUBSISTEMAS DE LA PLANTA PILOTO DE BIODIÉSEL

\begin{tabular}{|c|l|}
\hline Subsistema & \multicolumn{1}{|c|}{ Nombre del subsistema de la planta } \\
\hline 1 & Sistema de calentamiento de agua (CA) \\
\hline 2 & Sistema de reacción-reactor tipo Batch \\
\hline 3 & Sistema de alimentación del aceite usado (AU). \\
\hline 4 & Sistema de recuperación de alcohol (RA) \\
\hline 5 & Sistema de transesterificación y esterificación (STE) \\
\hline 6 & Sistema mezclador catalizador-alcohol (SC) \\
\hline 7 & Sistema de refrigeración (SR) \\
\hline
\end{tabular}

Fig. 4. PLANO DE MONTAJE DE LA PLANTA PILOTO DE 150 L/LOTE EN 3D (IZQ) - MONTAJE DE PLANTA DE BIODIÉSEL DE 150 L/LOTE EN EL EDIFICIO DE QUÍMICA DE LA UNIVERSIDAD NACIONAL DE COLOMBIA, SEDE BOGOTÁ (DER)

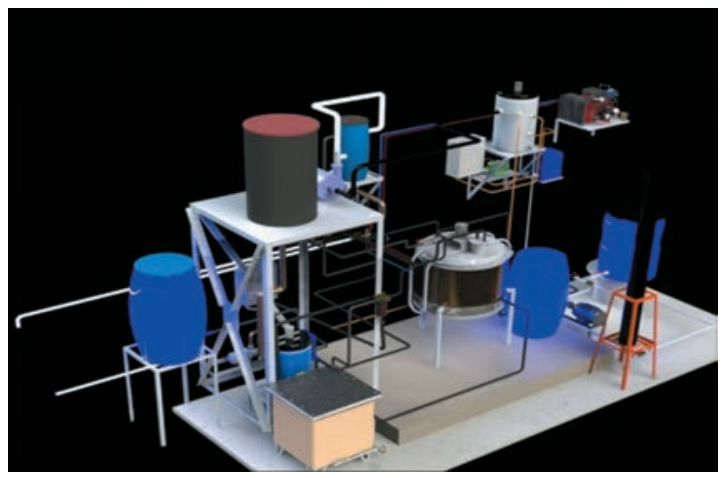

Fuente: autores.

\subsection{Pruebas con biocombustible en la caldera pirotubular}

Uno de los objetivos principales de esta investigación era el obtener un biodiésel que se implementará en equipos industriales -calderas-. Por lo cual se analizó el biodiésel obtenido en la planta piloto sin un postratamiento, es decir, un biodiésel de una menor calidad que cumple con algunos parámetros de la norma. Con base en esto se realizaron ensayos en la caldera vertical pirotubular de la Fig. 2, para identificar aspectos importantes, como el consumo, eficiencia energética y emisiones generadas. Para evaluar el consumo de la caldera se tomó el tiempo que dura el quemador prendido y el volumen de combustible alimentado antes de alcanzar la presión de operación (50 Psi), iniciando a una presión de 25 Psi la operación, los resultados se presentan en la Tabla IV.

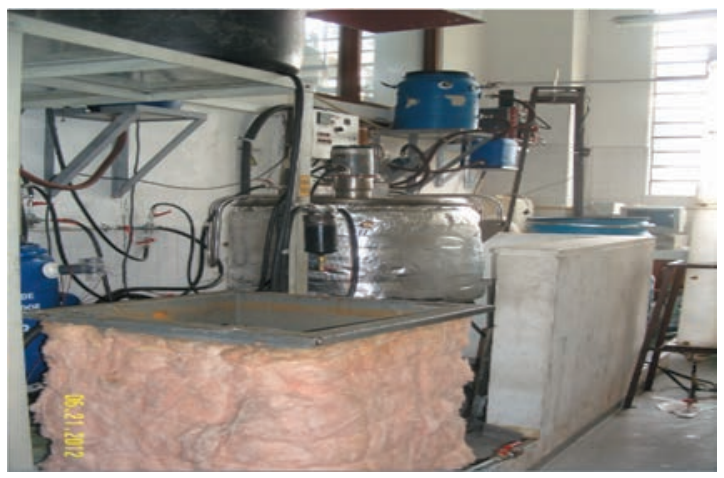

TABLA IV

ENSAYOS DE CONSUMO DE COMBUSTIBLE EN LA CALDERA VERTICAL PIROTUBULAR

\begin{tabular}{|c|c|}
\hline Ensayo & Consumo (L/min) \\
\hline 1 & 19,44 \\
\hline 2 & 31,57 \\
\hline 3 & 33,04 \\
\hline 4 & 17,76 \\
\hline 5 & 21,73 \\
\hline
\end{tabular}

Durante las pruebas realizadas a las 5 muestras de la combustión del biodiésel se encontró que el promedio del consumo estuvo alrededor de $24,71 \mathrm{~L} / \mathrm{min}$, asimismo, el biocombustible tiene una fácil ignición y mantiene una llama constante de color amarillo, condiciones que logran mantener una combustión uniforme en el quemador. Al obtener el set point de la caldera (40 psi) se obtienen temperaturas de $4^{\circ} \mathrm{C}$ (agua) - $37^{\circ} \mathrm{C}$ (vapor) 
a la entrada y $63^{\circ} \mathrm{C}$ (agua) $-77^{\circ} \mathrm{C}$ (vapor) en las salidas. En el caso de las emisiones generadas se empleó un analizador de gases marca Bacharach, modelo 300 con rangos de oxigeno $0-25 \% \mathrm{v} / \mathrm{v}$ y precisión de $+/-6 \%$, para el caso del monóxido de carbono tiene unos rangos de medición de 0 - 3000 ppm con una precisión de lectura del +/$5 \%$, con los datos obtenidos en el equipo se pudo establecer una reducción en el $\mathrm{CO}_{2}$ del 2,3\% y 84 ppm de $\mathrm{CO}$ entre las muestras analizadas de diésel y el biodiésel producido en la planta piloto. Estos aspectos permiten que el biodiésel elaborado en la planta piloto de 150L/Lote a partir de AUFS sea un combustible alternativo que pueda implementarse para su uso en calderas u otros equipos a nivel industrial, permitiendo una disminución en las emisiones de gases efecto invernadero, como en la dependencia con respecto a los combustibles fósiles.

\section{CONCLUSIONES}

Las propiedades del biodiésel son altamente influenciadas por la composición del índice de acidez (7,65 mg KOH/g aceite), viscosidad (25,65 $\mathrm{mm}^{2} / \mathrm{s}$ ) y densidad $921,5 \mathrm{Kg} / \mathrm{m}^{3}$ ) del aceite usado de fritura empleado como materia, porque a partir de la metodología de RSR mostraron más ventajas que las propiedades del biodiésel (Viscosidad $4,45 \mathrm{~mm} 2 / \mathrm{s}$, densidad $899 \mathrm{Kg} / \mathrm{m}^{3}$, índice de acidez 0,39 $\mathrm{mg} \mathrm{KOH} / \mathrm{g}$ aceite).

Los rendimiento obtenidos de los etil-ésteres de ácidos grasos según los análisis cromatográficos son del 96,4\%p/p, con unos rendimientos en peso del biodiésel con respecto a la cantidad inicial de aceite del $90 \%$ a nivel de laboratorio.

El biodiésel logrado en la planta piloto de 150L/Lote con AUFS y etanol a partir de los procesos de esterificación - transesterificación obtuvo un máximo rendimiento de 93,5\% de FAEE producido con una relación molar de 1:7 aceite: alcohol, $0,7 \% \mathrm{p} / \mathrm{p}$ de $\mathrm{NaOH}$, tiempo de reacción de 60 min, temperatura de reacción de $60^{\circ} \mathrm{C}$, catalizador el $\mathrm{NaOH}$ y velocidad de agitación de 200 RPM.

El producto obtenido posee características adecuadas para su uso como combustible en caldera, porque el comportamiento del biocombustibles logra y mantiene las condiciones de operación de la caldera para el uso en procesos industriales, y permite una disminución del 2,3\% de $\mathrm{CO}_{2}$.

\section{AGRADECIMIENTOS}

Los autores agradecen el apoyo de los grupos de investigación: Mecanismos de Desarrollo Limpio y Gestión Energética y Aprovechamiento Energético de Recursos Naturales, con el programa del Sistema de Información de la Investigación (Hermes) de la Universidad Nacional de Colombia por la financiación de proyecto 14354.

\section{REFERENCIAS}

[1] M. Leahy, J. L. Barden, B. T. Murphy, N. Slater-thompson, and D. Peterson, "International Energy Outlook 2013.", The U.S. Energy Information Administration (EIA), Washington DC. U.S, The annual report, pp. 55-87, Apr. 2013.

[2] M. Zabeti, W. M. A. Wan Daud, and M. K. Aroua, "Activity of solid catalysts for biodiesel production: A review," Fuel Process. Technol., vol. 90, no. 6, pp. 770-777, Jun. 2009.

[3] T. Abbasi and S. a. Abbasi, “Biomass energy and the environmental impacts associated with its production and utilization," Renew. Sustain. Energy Rev., vol. 14, no. 3, pp. 919-937, Apr. 2010.

[4] Y. M. Sani, W. M. A. W. Daud, and a. R. Abdul Aziz, "Activity of solid acid catalysts for biodiesel production: A critical review," Appl. Catal. A Gen., vol. 470, pp. 140161, Jan. 2014.

[5] C. A. Guerrero-Fajardo, Fabio Sierra-Vargas, Tecnologias para el aprovechamiento de los biocombustibles. Bogotá, Colombia, 2008, p. 326.

[6] M. K. Lam, K. T. Lee, and A. R. Mohamed, "Homogeneous, heterogeneous and enzymatic catalysis for transesterification of high free fatty acid oil (waste cooking oil) to biodiesel: a review.," Biotechnol. Adv., vol. 28, no. 4, pp. 500-18, 2010.

[7] A. E. Atabani, A. S. Silitonga, I. A. Badruddin, T. M. I. Mahlia, H. H. Masjuki, and S. Mekhilef, "A comprehensive review on biodiesel as an alternative energy resource and its characteristics," Renew. Sustain. Energy Rev., vol. 16, no. 4, pp. 2070-2093, May 2012.

[8] D. Y. C. Leung, X. Wu, and M. K. H. Leung, "A review on biodiesel production using catalyzed transesterification," Appl. Energy, vol. 87, no. 4, pp. 1083-1095, Apr. 2010.

[9] M. Berrios, M. A. Martín, A. F. Chica, and A. Martín, "Purification of biodiesel from used cooking oils," Appl. Energy, vol. 88, no. 11, pp. 3625-3631, Nov. 2011.

[10] J. M. Encinar, J. F. González, and A. Rodríguez-Reinares, "Ethanolysis of used frying oil. Biodiesel preparation and characterization," Fuel Process. Technol., vol. 88, no. 5, pp. 513-522, May. 2007. 
[11] J.-H. Tsai, S.-J. Chen, K.-L. Huang, Y.-C. Lin, W.-J. Lee, C.-C. Lin, and W.-Y. Lin, "PM, carbon, and PAH emissions from a diesel generator fuelled with soy-biodiesel blends.," J. Hazard. Mater., vol. 179, no. 1-3, pp. 237243, Jul. 2010.

[12] K. T. Tan, M. M. Gui, K. T. Lee, and A. R. Mohamed, "An optimized study of methanol and ethanol in supercritical alcohol technology for biodiesel production," J. Supercrit. Fluids, vol. 53, no. 1-3, pp. 82-87, Jun. 2010.

[13] F. Nacional de biocombustibles de Colombia, "Cifras informativas del sector biocombustibles-biodiésel de aceite de palma," vol. 1, p. 10, 2014.
[14] T. Issariyakul, M. G. Kulkarni, A. K. Dalai, and N. N. Bakhshi, "Production of biodiesel from waste fryer grease using mixed methanol/ethanol system," Fuel Process. Technol., vol. 88, no. 5, pp. 429-436, May. 2007.

[15] A. Hayyan, F. S. Mjalli, M. A. Hashim, M. Hayyan, I. M. Alnashef, S. M. Al-Zahrani, and M. A. Al-Saadi, "Ethanesulfonic acid-based esterification of industrial acidic crude palm oil for biodiesel production.," Bioresour. Technol., vol. 102, no. 20, pp. 9564-9570, Jul. 2011.

[16] Z. Yaakob, M. Mohammad, M. Alherbawi, Z. Alam, and K. Sopian, "biodiesel from Waste cooking oil," Renew. Sustain. Energy Rev., vol. 18, pp. 184-193, Feb. 2013. 